\title{
EDITORIAL
}

\section{Is heart rate variability the simple solution to diagnose sleep apnoea?}

\author{
T. Penzel
}

Early in the investigation of obstructive sleep apnoea it was recognised that the events of apnoea and hypopnoea are accompanied by concomitant cyclic variations in heart rate [1]. The pattern of brady/tachycardia is closely linked to the time course of apnoeic events. As a consequence, this pattern had been used successfully to detect sleep apnoea in patients with clinical symptoms for sleep apnoea. Dedicated devices were developed over the past 15 yrs to detect sleep apnoea at an early stage of the disease using recordings of heart rate, snoring, oxygen saturation and body position [2]. These devices were used successfully to stratify the individual risk of patients suffering from sleep-disordered breathing before they were referred to cardiorespiratory polysomnography. Sleep laboratories have limited capacity for cardiorespiratory polysomnography and are expensive in terms of personal costs due to continuous attendance during the night. The sensitivity and specifity of these simplified four channel-based recording systems had been evaluated in many studies giving results for specific systems. A recent systematic review compiled the results of these studies in a comparative overview [3]. The review revealed the value and the limitations of systems with respect to the accepted criteria for the diagnosis of sleep apnoea [4]. In conclusion, a satisfactory standardisation of terms and of diagnostic criteria regarding the portable diagnosis of sleep apnoea could not be achieved.

In parallel with efforts to define evidence-based criteria for the simplified diagnosis of sleep apnoea, the physiology causing the cyclic variation of heart rate was investigated. Studies on sympathetic neural activity during sleep apnoea proved that sympathetic activation increases during the course of the apnoea [5]. To what extent the peroneal nerve activity reflects sympathetic activation of the heart during apnoea, with effects on the heart rate itself, is not completely clear. The activation of sympathetic nerve activity in patients with sleep apnoea even persists during daytime and may contribute to the cardiovascular risk in these patients [6].

Two papers published in this issue investigate heart rate and heart rate variability (HRV) in sleep apnoea to improve means for a simplified diagnosis of disordered breathing during sleep [7, 8]. At the same time, both papers indicate that the physiological background of HRV is caused by sleep apnoea.

One of these investigations uses sympathetic activity derived from spectral components of HRV [7]. To derive indicators for sympathetic and parasympathetic tone from HRV by the calculation of spectral power in defined frequency bands is a well-established technique [9]. This technique proved to have some predictive value in hypertension and myocardial infarction and therefore it is now applied to many cardiovascular disorders. The low-frequency component of HRV is usually associated with sympathetic activity. The present study investigated spectral components in short 2-min time windows around apnoeas and compares

Correspondence: T. Penzel, Hospital of Philipps-University, Marburg, Germany. Fax: 49 64212865450. E-mail: penzel@mailer.uni-marburg.de them with undisturbed sleep in healthy control subjects. The article comes to the conclusion that there is an elevated sympathetic tone during disordered breathing [7].

Unfortunately, sleep-disordered breathing presents a complex interaction between the regular changes of the autonomous nervous system during sleep and the specific apnoea-related changes. Spectral analysis requires stationary signals in terms of physics. This means that the mean value and standard deviation and higher order moments of the signal analysed must remain the same for the period investigated. Sleep apnoea with its highly dynamic pattern of HRV is by no means stationary and therefore the application of spectral analysis is limited and corresponding results have to be interpreted with caution. The article discusses these inherent limitations and sets its results in a cautious context.

Due to these limitations other investigators try to apply new techniques especially developed for nonstationary data analysis. This has been done in the second article on this subject published in this issue [8]. The time-frequency wavelet analysis is a sophisticated technique superior to spectral analysis, since it is not restricted to the limiting stationary requirements. The difficulty with wavelet analysis is that the results are less intuitive and therefore an additional method must be applied that helps to select the wavelet that serves best to detect sleep apnoea. The investigation [8] has applied the wavelet method to a large sample of patients and has developed an optimal decision tree in order to identify the best wavelets for this purpose. The presented results prove that this method is very well suited to recognise the sleep apnoea-specific cyclic variability of heart rate, because the pattern is not strictly periodic. The application of this method can improve the recognition of sleep apnoea based on the noninvasive simple recording of electrocardiography (ECG) and heart rate.

Both studies confirm the finding that new methods can recognise sleep apnoea from HRV changes alone to a surprisingly good extent [10]. Thus, it seems to be possible to apply simplified recording techniques combined with sophisticated analysis techniques to detect sleep-disordered breathing. Latest advertisements of pacemakers do already offer an optional indication for the occurrence of sleep apnoea based on the analysis of past heart rate recordings as stored in their memory. Consequently, more cardiologists using long-term ECG recordings and using heart rate dumps from pacemakers may identify patients with suspected sleep apnoea.

The physiology behind the HRV changes are most interesting and few aspects of the heart-lung interactions had been clarified to date. Therefore, it is interesting to investigate the time course of sympathetic and parasympathetic activation during the event of apnoea and the following breaths itself [5]. The time course of sympathetic activation had been described by sympathetic nerve activity but the time course of parasympathetic activity had not been investigated. It is quite likely that both sympathetic and parasympathetic activity are increased at the end of each apnoea and then decline during the short period of compensatory hyperventilation. The 
methods to analyse these short-term effects based on HRV must take advantage of new algorithms that are not restricted to stationary signals, since this behaviour is very dynamic. Spectral analysis cannot be used for this specific purpose. A study to prove the concept of increased sympathetic and parasympathetic tone has not been conducted to date.

Heart rate does change with sleep stages, with disordered breathing during sleep and with disorders affecting the heart. New sophisticated methods may allow the separation of these influences and the separation of sleep stages, sleep apnoea and other disorders affecting the heart. Thus, the analysis of the easy accessible signal of ECG with its derived heart rate and HRV can serve numerous tasks. The decomposition of the multiple influences on ECG and heart rate to their original origins appears to be a promising vision. Today, this ultimate goal has not been reached due to the limitations of the analysis methods used.

To conclude, it has to be stated that surrogate parameters such as the brady/tachycardia pattern can never replace the target variables that are derived from the direct recording of respiration. Therefore, the derivation of an apnoea/hypopnoea index or a number of apnoea and hypopnoea events from heart rate variability analysis has to be viewed with great caution. As a consequence, derived measures and parameters should always be named differently to the apnoea/hypopnoea index to make the derived nature clear.

\section{References}

1. Guilleminault C, Winkle R, Connolly S, Melvin K, Tilkian A. Cyclical variation of heart rate in sleep apnea syndrome. Lancet 1984; i: $126-131$
2. Roos M, Althaus W, Rhiel C, Penzel T, Peter JH, von Wichert P. Comparative use of MESAM IV and polysomnography in sleep-related respiratory disorders. Pneumologie 1993; 47: Suppl. 1, 112-118.

3. Ross SD, Sheinhait IA, Harrison KJ, et al. Systematic review and meta-analysis of the literature regarding the diagnosis of sleep apnea. Sleep 2000; 23: 519-532.

4. American Academy of Sleep Medicine Task Force. Sleeprelated breathing disorder in adults: recommendations for syndrome definition and measurement techniques in clinical research. Sleep 1999; 22: 667-689.

5. Narkiewicz K, Somers VK. Sympathetic nerve activity in obstructive sleep apnoea. Acta Physiol Scand 2003; 177: 385390

6. Narkiewicz K, Pesek CA, Kato M, Phillips BG, Davison DE, Somers VK. Baroreflex control of sympathetic nerve activity and heart rate in obstructive sleep apnea. Hypertension 1998; 32: 1039-1043.

7. Dingli K, Assimakopoulos T, Wraith PK, Fietze I, Witt C, Douglas NJ. Spectral oscillations of RR intervals in sleep apnoea/hypopnoea syndrome patients. Eur Respir $J$ 2003; 22: 943-950.

8. Roche F, Pichot V, Sforza E, et al. Predicting sleep apnoea syndrome from heart period: a time-frequency wavelet analysis. Eur Respir $J$ 2003; 22: 937-942.

9. Task force of the European Society of Cardiology and the North American Society of Pacing and Electrophysiology. Heart rate variability. Standards of measurement, physiological interpretation, and clinical use. Circulation 1996; 93: 1043-1065.

10. Penzel T, McNames J, de Chazal P, Raymond B, Murray A, Moody G. Systematic comparison of different algorithms for apnoea detection based on electrocardiogram recordings. Med Biol Eng Comput 2002; 40: 402-407. 